\title{
PAPER
}

\section{Dietary treatment of gluten ataxia}

\section{Hadjivassiliou, G A B Davies-Jones, D S Sanders, R A Grünewald}

J Neurol Neurosurg Psychiatry 2003;74:1221-1224

See end of article for authors' affiliations

......................

Correspondence to: Dr M Hadjivassiliou, Department of Clinical Neurology, The Royal Hallamshire Hospital, Glossop Road, Sheffield S10 2JF, UK;

m.hadjivassiliou@ sheffield.ac.uk

Received

7 January 2003

In revised form

19 February 2003

Accepted

23 February 2003
Background: Gluten ataxia is an immune mediated disease, part of the spectrum of gluten sensitivity, and accounts for up to $40 \%$ of cases of idiopathic sporadic ataxia. No systematic study of the effect of gluten-free diet on gluten ataxia has ever been undertaken.

Objective: To study the effect of gluten-free diet on patients presenting with ataxia caused by gluten sensitivity.

Methods: 43 patients with gluten ataxia were studied. All were offered a gluten-free diet and monitored every six months. All patients underwent a battery of tests to assess their ataxia at baseline and after one year on diet. Twenty six patients (treatment group) adhered to the gluten-free diet and had evidence of elimination of antigliadin antibodies by one year. Fourteen patients refused the diet (control group). Three patients had persistently raised antigliadin antibodies despite adherence to the diet and were therefore excluded from the analysis.

Results: After one year there was improvement in ataxia reflected in all of the ataxia tests in the treatment group. This was significant when compared with the control group. The diet associated improvement was apparent irrespective of the presence of an enteropathy.

Conclusions: Gluten ataxia responds to a strict gluten-free diet even in the absence of an enteropathy. The diagnosis of gluten ataxia is vital as it is one of the very few treatable causes of sporadic ataxia.
G luten ataxia ${ }^{1}$ is an immunologically mediated disease triggered by the ingestion of gluten in genetically susceptible individuals. Gluten ataxia forms part of a spectrum of disorders associated with gluten sensitivity which include coeliac disease (gluten sensitive enteropathy) and dermatitis herpetiformis (gluten sensitive dermatopathy).

An epidemiological study of the prevalence of gluten ataxia suggested that it may account for up to $40 \%$ of patients with sporadic idiopathic ataxia. ${ }^{2}$ Seventy two per cent of these patients have the HLA DQ2 antigen, which is found in $90 \%$ of patients with both gluten sensitive enteropathy and dermatitis herpetiformis. ${ }^{2}$ Gluten ataxia is the commonest single cause of cerebellar ataxia among patients with supposed idiopathic sporadic ataxia.

The benefits of a gluten-free diet in the treatment of patients with coeliac disease and dermatitis herpetiformis have long been established. ${ }^{3}$ There are very few studies (mainly case reports) of the effect of gluten-free diet on the neurological manifestations of gluten sensitivity. ${ }^{4-10}$ All but one of these reports primarily concern patients with established coeliac disease who then develop neurological symptoms. A small uncontrolled study looked at the use of intravenous immunoglobulins in the treatment of four patients with gluten ataxia without enteropathy. ${ }^{11}$ All four patients improved, confirming the contention that gluten ataxia is immune mediated. There was no mention, however, that dietary treatment had been considered and assessed before the use of immunomodulatory therapy.

Overall, these studies suggest variable responsiveness to treatment. In some case reports, adherence to a gluten-free diet is assumed or based on improvement of gastrointestinal symptoms or on duodenal biopsy, without concurrent serological evidence of elimination of circulating antigliadin antibodies. No systematic study of the effect of a gluten-free diet on a cohort of patients presenting with neurological dysfunction with or without an enteropathy has yet been reported.

Our first aim in this study was to establish any therapeutic effects of the gluten-free diet in patients with gluten sensitivity presenting with ataxia. Our second aim was to provide fur- ther evidence, by studying patients with gluten ataxia but without enteropathy, that the nervous system can be the sole target organ of an immune mediated disease triggered and perpetuated by the ingestion of gluten.

\section{METHODS}

\section{Patient selection}

Patients with gluten ataxia (idiopathic sporadic ataxia with positive antigliadin antibodies) were identified and studied between 1996 and 2003. The study protocol was approved by the South Sheffield research ethics committee and all patients gave their written informed consent.

Other causes of ataxia were excluded by extensive investigation, including magnetic resonance imaging of the brain, genetic testing for spinocerebellar ataxias 1, 2, 3, 6, and 7 and Freidreich's ataxia, estimation of serum vitamin E, and thyroid function tests. Additional tests were done if clinically indicated. Patients with a history of alcohol abuse, prolonged use of the anticonvulsant drug phenytoin, multiple sclerosis, vitamin E deficiency, paraneoplastic cerebellar degeneration, and viral cerebellitis were excluded.

All patients underwent duodenal biopsy and those with or without an enteropathy (defined as villous atrophy, crypt hyperplasia, and increased intraepithelial lymphocytes) were included in the study. We devised a pragmatic cohort study in which all patients with gluten ataxia were offered a gluten-free diet. We compared the neurological response in those who adhered to the diet with those who refused the diet, estimating compliance with the diet in both groups by antigliadin antibody titres. Detailed dietary advice by a dietician expert in gluten sensitivity was given to all the patients. The gluten-free diet was started after baseline assessment of the ataxia. The patients were reviewed at six monthly intervals by both a neurologist and a dietician, but had access to dietary advice over the telephone at any time during the period of the study. The assessment of ataxia was repeated after a year of strict adherence to the diet, confirmed by serological evidence of elimination of circulating antigliadin antibodies (in our experience antigliadin antibodies 
persist for up to six months after the introduction of a glutenfree diet). Patients with gluten ataxia who refused the diet were followed up in an identical manner, with demonstration of persistently positive antigliadin antibodies in their sera.

\section{Assessment of ataxia}

Clinical assessment of the patients was undertaken by one investigator $(\mathrm{MH})$, thus minimising interobserver variability. Although he was not blinded to the dietary compliance of the patients, he was not aware of the results of antigliadin antibody testing at the time of the one year assessments. In addition, all the one year assessments took place without knowledge of the baseline results.

There are very few ataxia assessment scales available and none has been validated. We have selected tests based on the standard neurological examination which had been validated in patients with progressive ataxia, and are as far as possible objective. ${ }^{12}$ The accuracy of recording of latency for the fingernose test was enhanced by the use of a computer with a touch sensitive screen. The tests were as follows:

\section{Computerised finger nose test}

This was done by adapting the motor screening test from the Cambridge automated neuropsychological test battery (CANTAB).${ }^{13}$ Patients were placed at arm's length from a touch sensitive screen. They were asked to place their right index finger on the tip of their nose and instructed to touch, as quickly and accurately as possible, the centre of a flashing cross that appeared on the screen. As soon as the patient touched the cross the image disappeared. Patients were instructed to place their finger back on the tip of their nose immediately until the image reappeared on the screen. The image reappeared nine times at different positions on the screen but at the same time interval. The test was repeated using the left index finger. The computer recorded the mean response latency (in milliseconds).

\section{Grooved pegboard test (Lafayette Instruments, Sagamore Parkway, Indiana, USA)}

This is a test of manipulative dexterity that requires more complex motor coordination than most pegboards. The board consisted of 25 holes with randomly positioned locating slots. The pegs, which have a key along one side, must be rotated to match the slot position before they can be inserted. The subjects were asked to insert the pegs starting from the top and from right to left using only one hand and without additional help from the other hand, as fast as possible. The time taken to fill all the holes was recorded. The task was repeated using the left hand.

\section{Tapping test}

The test uses a device consisting of two manually operated counters fixed $35 \mathrm{~cm}$ apart on a wooden platform. The patienta are placed behind a desk with the device positioned at arm's length. They are then asked to press with their index finger the left and then the right button of the counter alternately as fast as they can for 30 seconds. The task is repeated using the left hand. The total count from the two counters after 30 seconds was recorded. The same device placed on the floor at a fixed distance away from each front leg of the chair was then used for each foot in turn.

\section{Quantitative Romberg's test}

Patients were asked to stand with feet together and eyes closed. They were instructed to try to keep their eyes closed and their feet together as long as possible. The time to first corrective feet movement or eye opening was recorded. This test was maximally sensitive only in subjects with moderate ataxia. Because of pronounced "floor" and "ceiling" effects in this test (that is, some patients were unable to attempt the
Table 1 Clinical characteristics of the gluten ataxia treatment and control groups

\begin{tabular}{|c|c|c|}
\hline & $\begin{array}{l}\text { GA treatment } \\
\text { group }\end{array}$ & GA control group \\
\hline$n$ & 26 & 14 \\
\hline Male:female & $20: 6$ & $11: 3$ \\
\hline Mean age (years) (range) & 63 (28 to 84 ) & 63 (38 to 82 ) \\
\hline $\begin{array}{l}\text { Duration of ataxia (years) } \\
\text { (range) }\end{array}$ & $9(2$ to 25$)$ & 11 (2 to 27$)$ \\
\hline Enteropathy on biopsy & 8 & 1 \\
\hline
\end{tabular}

test, others could stand indefinitely), changes in performance during the study were recorded as or "better" or "worse or same" and analysed non-parametrically.

\section{Subjective global clinical impression}

Patients were asked to mark on a visual analogue scale their impression of their symptoms of imbalance over the last month. No change was marked at the centre of the line, better towards the right end of the line, and worse towards the left end. The distance from the left end of the line to the mark made by the patient was recorded as a proportion of the total length of the line.

\section{Statistical analysis}

In those tests where measurements were made from both left and right sides or the body independently, the summed data from both sides were analysed statistically. Continuous variables were compared at baseline and after one year on diet by repeated measures analysis of variance. The quantitative Romberg's test results, converted to categories "improved" or "worse or same," were compared in the experimental and control groups by the $\chi^{2}$ test. Statistical analysis was undertaken using the statistical package GB-Stat version 6.5 (Dynamic Microsystems Inc, Silver Spring, Maryland, USA).

\section{RESULTS}

Forty three patients with gluten ataxia were enrolled in the study. Twenty six adhered strictly to the gluten-free diet and had serological evidence of elimination of the antigliadin antibodies. These patients comprised the treatment group. Fourteen refused the diet and comprised the control group. Three claimed to have adhered to the gluten-free diet but had persistently positive antigliadin antibodies and were excluded from the analysis. All patients attended for the six month and one year assessments.

Table 1 summarises the characteristics of the two groups. The mean age for both the treatment and control groups was 63 years. The mean duration of ataxia was nine years in the treatment group and 11 years in the control group. Eight of 26 patients in the treatment group and one of 14 in the control had duodenal biopsies in keeping with coeliac disease. The clinical picture was identical in patients with or without an enteropathy. Twenty three patients in the treatment group had positive IgG antigliadin antibodies. Eight had IgA antigliadin antibodies and five had both. All of the 14 patients in the control group had IgG antigliadin antibodies. Four also had IgA antigliadin antibodies. Patient and control groups were matched at baseline for performance in all evaluated variables.

Table 2 summarises the results of the ataxia assessment at baseline and at one year for the treatment and control groups. There was a significant improvement in performance in all the tests and in the subjective global clinical impression scale in the treatment group when compared with the control group. The improvement in all the tests in the treatment group was also apparent after excluding patients with an enteropathy (table 3 ). When the three patients with persistently positive 
Table 2 Results of ataxia tests when comparing the treatment and the control groups

\begin{tabular}{|c|c|c|c|c|c|}
\hline \multirow[b]{2}{*}{ Test } & \multicolumn{2}{|c|}{ GA treatment group $(n=26)$} & \multicolumn{2}{|c|}{ GA control group $(n=14)$} & \multirow{2}{*}{$\begin{array}{l}p \text { Value of } \\
\text { interaction }\end{array}$} \\
\hline & Baseline & One year & Baseline & One year & \\
\hline $\begin{array}{l}\text { Computerised finger-nose test latency } \\
\text { (ms) }\end{array}$ & 3296 (1133) & 2503 (717) & $3372(1694)$ & $3716(2066)$ & 0.0001 \\
\hline Hand tapping & $80(32)$ & $89(33)$ & $59(28)$ & 50 (19) & 0.0002 \\
\hline Foot tapping & 79 (37) & $95(41)$ & $78(29)$ & $72(26)$ & 0.0001 \\
\hline Peg board, time to fill (s) & $291(146)$ & $248(80)$ & $410(201)$ & $496(292)$ & 0.0015 \\
\hline Quantitative Romberg's test & \multicolumn{2}{|c|}{18 of 26 patients improved } & \multicolumn{2}{|c|}{ None of 14 improved } & 0.0001 by $\chi^{2}$ test \\
\hline Patients' global clinical impression & $0.41(0.18)$ & $0.62(0.25)$ & $0.39(0.15)$ & $0.31(0.14)$ & 0.0002 \\
\hline
\end{tabular}

Values are mean (SD).

Statistical analysis is by repeated measures analysis of variance except where stated.

GA, gluten ataxia.

\begin{tabular}{|c|c|c|c|c|c|}
\hline \multirow[b]{2}{*}{ Test } & \multicolumn{2}{|c|}{ GA treatment group $(n=18)$} & \multicolumn{2}{|c|}{ GA control group $(n=13)$} & \multirow{2}{*}{$\begin{array}{l}\text { - V Value of } \\
\text { interaction }\end{array}$} \\
\hline & Baseline & One year & Baseline & One year & \\
\hline Computerised finger-nose test latency (ms) & 3107 (1089) & $2350(605)$ & $3199(1630)$ & 3485 (1952) & 0.0005 \\
\hline Hand tapping & $81(27)$ & $92(32)$ & $61(28)$ & $52(20)$ & 0.0003 \\
\hline Foot tapping & $79(27)$ & $97(34)$ & $82(25)$ & $75(23)$ & 0.0001 \\
\hline Peg board, time to fill (s) & $298(158)$ & $243(65)$ & $401(206)$ & $471(288)$ & 0.0094 \\
\hline Quantitative Romberg's test & \multicolumn{2}{|c|}{14 of 18 patients improved } & \multicolumn{2}{|c|}{ None of 13 improved } & 0.0001 by $\chi^{2}$ test \\
\hline Patients' global clinical impression & $0.4(0.1)$ & $0.62(0.24)$ & $0.40(0.15)$ & $0.31(0.15)$ & 0.0004 \\
\hline
\end{tabular}

Values are mean (SD).

Statistical analysis is by repeated measures analysis of variance except where stated.

GA, gluten ataxia.

antigliadin antibodies despite gluten-free diet were included in the analysis (in the treatment group) the significant difference persisted.

\section{DISCUSSION}

Our previous finding of a high prevalence of gluten sensitivity in patients with otherwise unexplained neurological illness ${ }^{14}$ (particularly ataxia and peripheral neuropathy) resulted in a debate as to whether neurological illness is associated with, rather than a manifestation of, sensitivity to gluten. The evidence that gluten ataxia is a manifestation of gluten sensitivity is now substantial and analogous to the example of dermatitis herpetiformis, from which it is apparent that the gut is not the sole protagonist in this disease. Dermatitis herpetiformis is best described as a gluten sensitive dermatopathy triggered and perpetuated by gluten and thus responsive to a gluten-free diet. The majority but not all patients with dermatitis herpetiformis have an enteropathy on duodenal biopsy, yet in contrast to coeliac disease, gastrointestinal symptoms are rarely the presenting feature.

Neuropathological data from patients with gluten ataxia confirm that inflammation is a prominent feature in the pathogenesis of this condition, in keeping with the model of an immune mediated disease. The presence of circulating antigliadin antibodies (which define gluten ataxia) is not a non-specific finding as it is accompanied by a strong HLA association similar to that seen in patients with coeliac disease (DQ2). ${ }^{2}$

Further compelling evidence for an immune pathogenesis comes from research showing the presence of circulating Purkinje cell antibodies in the serum of patients with gluten ataxia. ${ }^{15}$ The same research has shown that antigliadin antibodies cross react with Purkinje cells, suggesting the sharing of common epitopes between gliadin proteins and Purkinje cells.

In this paper we report improvement in the ataxia associated with adherence to a gluten-free diet. This confirms our contention that gluten ataxia is another manifestation of, and not merely an association with, gluten sensitivity. Furthermore the finding of improvement of the ataxia in patients without an enteropathy suggests that other organ specific manifestations can be triggered and perpetuated by the ingestion of gluten in the absence of an enteropathy.

The design of this study posed considerable challenges. The onset of this study in 1996 followed on from the identification and description of gluten ataxia by our group. ${ }^{1}$ At the time, information on the prevalence of this disorder was limited. In addition the prevalence of an enteropathy among patients with gluten ataxia was also unclear. Randomising patients with gluten ataxia to treatment or not with gluten-free diet would have required the exclusion of patients with an enteropathy or who had developed symptoms or pathological evidence of an enteropathy during the study. Furthermore, if patients were assigned to the control group they would inevitably avoid diets rich in gluten (for example, bread and pasta) despite advice to the contrary (unpublished observation). This would further dilute possible differences in response between the two groups. On clinical grounds-both neurological and gastrointestinal-we were unable to distinguish between patients with gluten ataxia with or without an enteropathy, as gastrointestinal symptoms were inconspicuous. In line with patient preference, we therefore devised a pragmatic cohort study in which all patients with gluten ataxia were offered a gluten-free diet. We compared the neurological response in those who adhered to the diet with those who refused the diet, estimating compliance with the diet by means of antigliadin antibody titres.

Such a study design may be associated with bias-for example, patients may be more likely to default from the diet if they see no immediate improvement in their symptoms. However, we emphasised to patients that improvement, if at all, on diet was likely to be slow and to take several months. The groups were well matched for severity and type of syptom.

An additional source of bias was that one investigator undertook all clinical assessments and was not blinded to the 
treatment state of the patients. This bias was minimised as far as possible by the use of objective ataxia scales and by his remaining blinded to the results of antigliadin antibody estimation and baseline ataxia assessment results when testing was undertaken at one year.

Neurophysiological assessment of peripheral nerve function in patients with gluten ataxia and peripheral neuropathy also showed improvement, supporting our contention that the improvement in the group adhering to the gluten-free diet was real. ${ }^{16}$ The neuropathological finding of loss of Purkinje cells in patients with gluten ataxia leads us to believe that intervention would probably result in stabilisation rather than improvement of the ataxia. That this was not the case and that ataxia improved in the treatment group is somewhat surprising. The improvement occurred irrespective of the duration of the ataxia, though some patients with a shorter duration of ataxia returned to normality. The results suggest that before the loss of Purkinje cells, there is a reversible process, perhaps related to active inflammation causing cerebellar dysfunction. Such inflammation has been described at necropsy in patients with gluten ataxia. ${ }^{1}$ This is analogous to other inflammatory cerebellar diseases such as paraneoplastic cerebellar degeneration, where removal of the cancer may result in gain of function, and postviral cerebellitis, where spontaneous recovery is the rule.

One of the patients with gluten ataxia in the control group who had a normal duodenal biopsy subsequently developed mild anaemia and positive serology for both antiendomysium and transglutaminase antibodies, suggestive of an enteropathy. This implies that patients with gluten ataxia without an enteropathy may well have latent coeliac disease. ${ }^{17}$ Another patient in the control group who had the ataxia for 22 years died recently from a high grade lymphoma, a well recognised complication of untreated gluten sensitive enteropathy. His duodenal biopsy before enrolment in the study showed no evidence of an enteropathy. A third patient in the control group developed cerebellar atrophy within 15 months of the onset of his ataxia. These observations pose serious ethical issues regarding randomisation of patients with gluten ataxia to no intervention.

Both gastroenterologists and neurologists need to be aware of the spectrum of gluten sensitivity with its many diverse manifestations. ${ }^{18}$ The erroneous belief that gluten sensitivity is exclusively a disease of the gut is detrimental to those patients presenting with neurological manifestations, as the absence of symptoms or histological evidence of enteropathy sometimes prevents them from receiving treatment with a gluten-free diet. One may argue that there is no longer a need for a small bowel biopsy in patients presenting with gluten ataxia or dermatitis herpetiformis, given that a gluten-free diet should be recommended irrespective of the state of the gut. Alternatively, given the diversity of organ involvement in this disease, precise characterisation of each group (with small bowel biopsies) may help in the understanding of the pathophysiology and the degree of overlap of gluten related diseases.

\section{Conclusions}

Gluten-free diet appears to be an effective treatment for gluten ataxia. It is imperative, however, that close monitoring should be undertaken with the use of antigliadin antibodies and dietetic review to ensure strict adherence to the diet. Immune treatment with immunosuppressants and intravenous immunoglobulins should be considered only if a strict gluten-free diet for at least a year has not resulted in any improvement in the ataxia or if the ataxia is rapidly progressive. The diagnosis of gluten ataxia is vital, as it is one of the very few treatable causes of sporadic ataxia.

\section{ACKNOWLEDGEMENTS}

We thank Julia Selby for providing all the dietetic advice and monitoring the diet of patients with gluten ataxia, and the Ataxia UK Group for funding aspects of our work into gluten ataxia.

\section{Authors' affiliations}

M Hadjivassiliou, G A B Davies-Jones, R A Grünewald, Department of Clinical Neurology, The Royal Hallamshire Hospital, Sheffield, UK D S Sanders, Department of Gastroenterology, The Royal Hallamshire Hospital

Competing interests: none declared

\section{REFERENCES}

1 Hadiivassiliou M, Grünewald RA, Chattopadhyay AK, et al. Clinical, radiological, neurophysiological and neuropathological characteristics of gluten ataxia. Lancet 1998;352:1582-5.

2 Hadjivassiliou M, Grünewald RA, Sharrack B, et al. Gluten ataxia in perspective: epidemiology, genetic susceptibility and clinical characteristics. Brain 2003; 126:685-91.

3 Ciclitira PJ, King AL, Fraser J. AGA technical review on celiac sprue. Gastroenterology 2001:120:1526-40.

4 Ward ME, Murphy JT, Greenberg GR. Coeliac disease and spinocerebellar degeneration with normal vitamin E status. Neurology 1985;35:1199-201

5 Kaplan JG, Pack D, Horoupian D, et al. Distal axonopathy associated with chronic gluten enteropathy: a treatable disorder. Neurology 1988;38:642-5.

6 Mauro A, Orsi L, Montara P, et al. Cerebellar syndrome in adult celiac disease with vitamin E deficiency. Acta Neurol Scand 1991;84:167-70.

7 Hermaszewski RA, Rigby S, Dalgleish AG. Coeliac disease presenting with cerebellar degeneration. Postgrad Med J 1991;67:1023-4.

8 Bhatia KP, Brown P, Gregory R, et al. Progressive myoclonic ataxia associated with coeliac disease: the myoclonus is of cortical origin, but the pathology is in the cerebellum. Brain 1995;118:1087-93.

9 Muller AF, Donnelly MT, Smith CML, et al. Neurological complications of coeliac disease: a rare but continuing problem. Am J Gastroenterol 1996:91:1430-5

10 Pellecchia MT, Scala R, Perretti A, et al. Cerebellar ataxia associated with subclinical celiac disease responding to gluten-free diet. Neurology 1999;53:1606-7

11 Bürk K, Melms A, Schulz JB, et al. Effectiveness of intravenous immunoglobulin therapy in cerebellar ataxia associated with gluten sensitivity. Ann Neurol 2001;50:827-8.

12 Notemans NC, Van Dijk GW, Van der Graaf Y, et al. Measuring ataxia: quantification based on the standard neurological examination. $J$ Neurol Neurosurg Psychiatry 1994;57:22-6.

13 Sahakian BJ, Owen AM. Computerised assessment in neuropsychiatry using CANTAB. J R Soc Med 1992;85:399-402.

14 Hadjivassiliou $M$, Gibson A, Davies-Jones GAB, et al. Is cryptic gluten sensitivity an important cause of neurological illness? Lancet 1996:347:369-71.

15 Hadiivassiliou M, Boscolo S, Davies-Jones A, et al. The humoral response in the pathogenesis of gluten ataxia. Neurology 2002;58: 1221-6.

16 Hadjivassiliou M, Grünewald RA, Chattopadhyay AK, et al. Gluten ataxia and gluten neuropathy: the effect of gluten fee diet. J Neurol Neurosurg Psychiatry 2002;72:139.

17 Ferguson A, Arranz E, O'Mahony S. Clinical and pathological spectrum of coeliac disease - active, silent, latent, potential. Gut 1993;34:150-1.

18 Hadjivassiliou M, Grünewald RA, Davies-Jones GAB. Gluten sensitivity as a neurological illness. J Neurol Neurosurg Psychiatry 2002;72:560-3. 